\title{
Surgical treatment of an aneurysm involving ascending aorta, aortic arch, and a rupture of a descending aortic aneurysm 26 years following acute type $A$ aortic dissection
}

\author{
Christian Kühn, MD, ${ }^{a}$ Omke E. Teebken, MD, ${ }^{a}$ Jürgen Weidemann, MD, ${ }^{\mathrm{b}}$ Matthias Karck, MD, ${ }^{\text {a }}$ \\ Maximillian Pichlmaier, MD, and Axel Haverich, MD, ${ }^{a}$ Hannover, Germany
}

\section{Supplemental material is available online.}

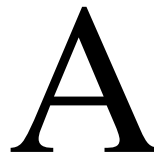

60-year-old man with distal aortic ascending aneurysm, aortic arch aneurysm and subacute rupture of the descending aorta had undergone aortic valve repair and proximal ascending aorta replacement following acute type A aortic dissection 26 years ago. Surgical therapy included replacement of distal ascending, arch, and complete thoracoabdominal descending aorta using selective antegrade cerebral and abdominal organ perfusion, reinsertion of the brachiocephalic trunc, left carotid artery, two intercostal arteries (Th-9/10), reimplantation of both renal arteries, the superior mesenteric artery and coeliac trunc through a left-sided thoracotomy and thoracoabdominal approach.

\section{Clinical Summary}

A patient underwent emergency aortic valve reconstruction combined with proximal ascending aorta replacement following traumatic type A aortic dissection after a ski-accident 26 years ago. The following years were uneventful and the patient was in good condition without signs of kidney dysfunction or abdominal malperfusion. An increasing aneurysm (diameter $4,5 \mathrm{~cm}$ ) of the left common iliac artery was resected and a $10 \mathrm{~mm}$ Dacron tube graft inserted two years ago. A computed tomography scan one year later showed the known dissection of the aortic arch and descending aorta (Figure 1). However, diameter of the aortic arch and the descending aorta had increased to $6.5 \mathrm{~cm}$ and $7.5 \mathrm{~cm}$, respectively just proximal to the diaphragm. In addition, a contained rupture was apparent.

\section{Surgical Technique}

In the right recumbent position, a skin incision was made from the lower angulus of the left scapula down to the belly button. The

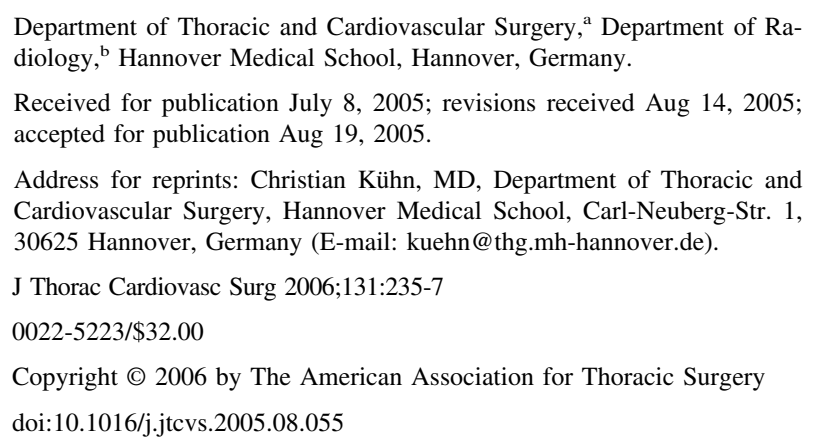

fourth and the eighth intercostal spaces were opened, the costal arch was divided, succeded by preparation of the retroperitoneum after incision of the diaphragm. Cardiopulmonary bypass was established through cannulation of the left femoral artery and vein. During induction of hypothermia $\left(26^{\circ} \mathrm{C}\right)$, the descending aorta (including the area of rupture), the aortic arch and the ascending aorta were dissected (Figure E1). Following onset of ventricular fibrillation the descending aorta was clamped. Meanwhile perfusion of the lower body was maintained by extracorporal circulation, the aortic arch was opened and selective cerebral perfusion was established via balloon tipped catheter in the brachiocephalic trunk and the left carotid artery. The left subclavian artery was clamped. Cardioplegic arrest was induced using a modified large ballon catheter for occlusion of the ascending aorta and administration of cardioplegia solution. An end-to-end anastomosis of the in situ ascending Dacron graft and a $28 \mathrm{~mm}$ woven Dacron graft was performed. The brachiocephalic trunk and the left carotid artery were reinserted as an island cuff. Selective cerebral perfusion was stopped, and retrograde perfusion of the upper body via cannulation of the prosthesis was reestablished. Following reinsertion of two intercostal arteries (Th-9 and Th-10), the heavily calcified infrarenal aorta was dissected and after discontinuation of lower body bypass it was anastomosed in end-to-end technique to a second 28-mm woven Dacron graft. Meanwhile, the abdominal organ perfusion was secured via balloon tipped catheters in the superior mesenteric artery, coeliac trunc and the left renal artery. The reimplantation of these vessels and the right renal artery was accomplished as an island cuff. A final end-to-end graft-to-graft anastomosis and removal of perfusion catheters completed the aortic repair. During rewarming, a side-to-end bypass between the distal aortic arch graft and the left subclavian artery was performed. The patient was weaned from cardiopulmonary bypass after 333 minutes bypass time with minimal inotropic support. The further postoperative course was uneventful in the absence of paraplegia and cognitive deficits and the patient was discharged in good conditions on postoperative day 13 .

\section{Discussion}

The multiple pathology in this patient including aortic arch aneurysm, chronic dissection of a distal aortic ascending aneurysm and a rupture of the enlarged descending aorta twenty-six years after traumatic type A aortic dissection with surgical aortic valve repair and proximal ascending aortic replacement posed a tactical and surgical challenge. We decided to replace the entire aorta including the distal ascending aorta, aortic arch and the thoracoabdominal descending aorta in one session $^{1,2}$. Therefore, the patient underwent a single-stage procedure 

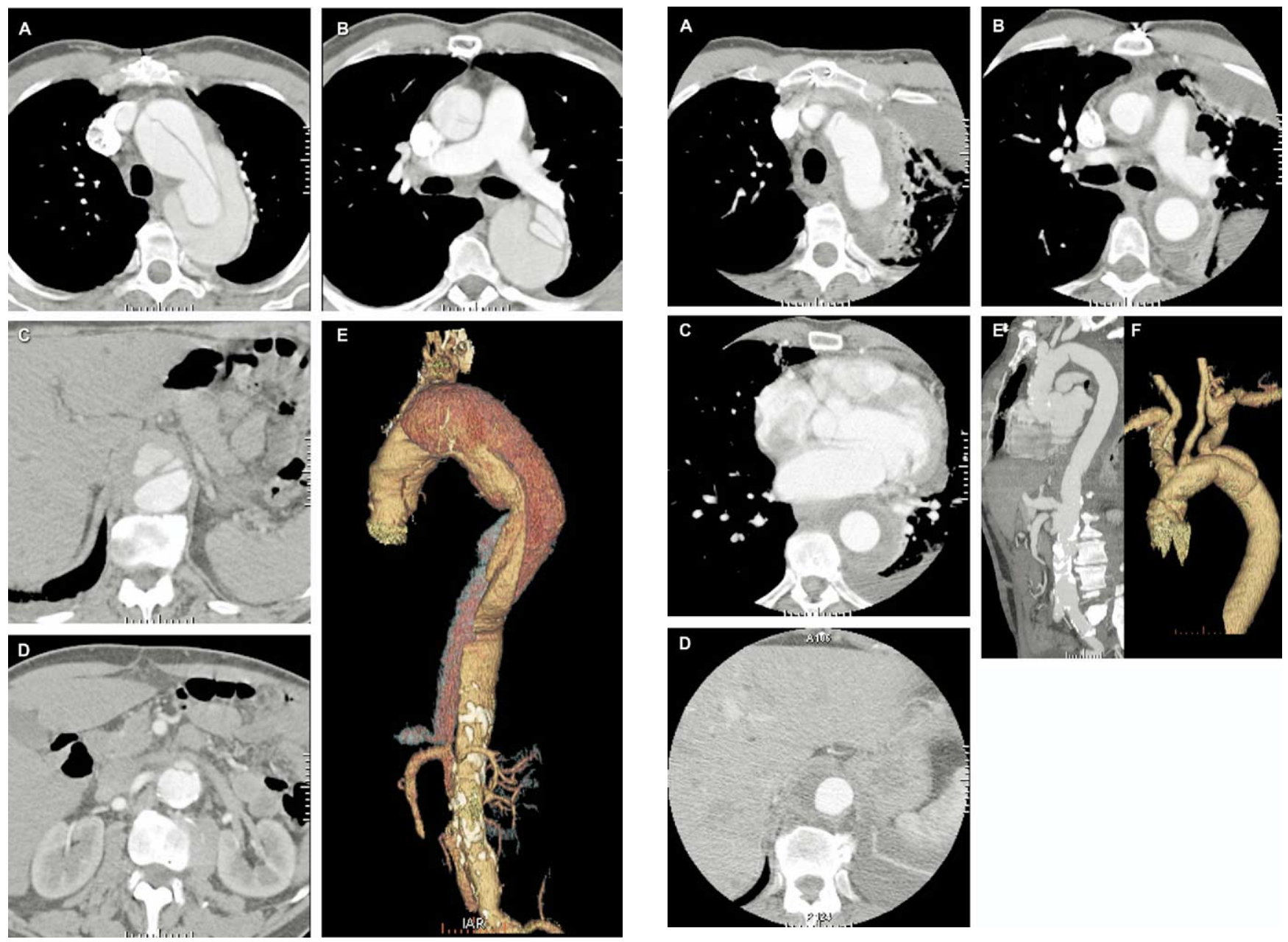

Figure 1. Preoperative computed tomography scan showing the complex aortic dissection involving arch (a), descending (b), thoraco-abdominal (c) and perirenal (d) aorta. The coloured reconstruction shows (e) the various lumens, however, the contained rupture is only apparent in (c).

through a thoracoabdominal approach combined with left thoracotomy. In hypothermia we used selective cerebral perfusion and abdominal organ perfusion for organ protection ${ }^{3}$. The patient was discharged without neurological deficits or signs for malperfusion with good liver and kidney function and went back to normal life. The postoperative computed tomography (Figure 2) showed a satisfactory result of the entire aorta after the approach that is described in this report as a useful treatment alternative in patients with these rare combination of diagnoses.

Figure 2. Postoperative computed tomography scan after replacement of the entire aorta including the distal ascending aorta (b), aortic arch (a), descending (b, c) and the thoraco-abdominal (d) aorta; oblique reformation (e) and coloured reconstruction (f).

\section{References}

1. Tanaka H, Yamashita T, Okada K, Okita Y. Successful surgery in a patient with a rupture of descending aorta complicated by acute type A aortic dissection through left-sided thoracotomy. Interact CardioVasc Thorac Surg. 2005;4:116-7.

2. Beaver TM, Martin TD. Single-stage transmediastinal replacement of the ascending, arch, and descending aorta. Ann Thorac Surg. 2001;72: 1232-8.

3. Hagl C, Khaladj N, Karck M, Kallenbach K, Leyh R, Winterhalter M, et al. Hypothermic circulatory arrest during ascending and aortic arch surgery: the theoretical impact of different cerebral perfusion techniques and other methods of cerebral protection. Eur J Cardiothorac Surg. 2003; 24:371-8. 

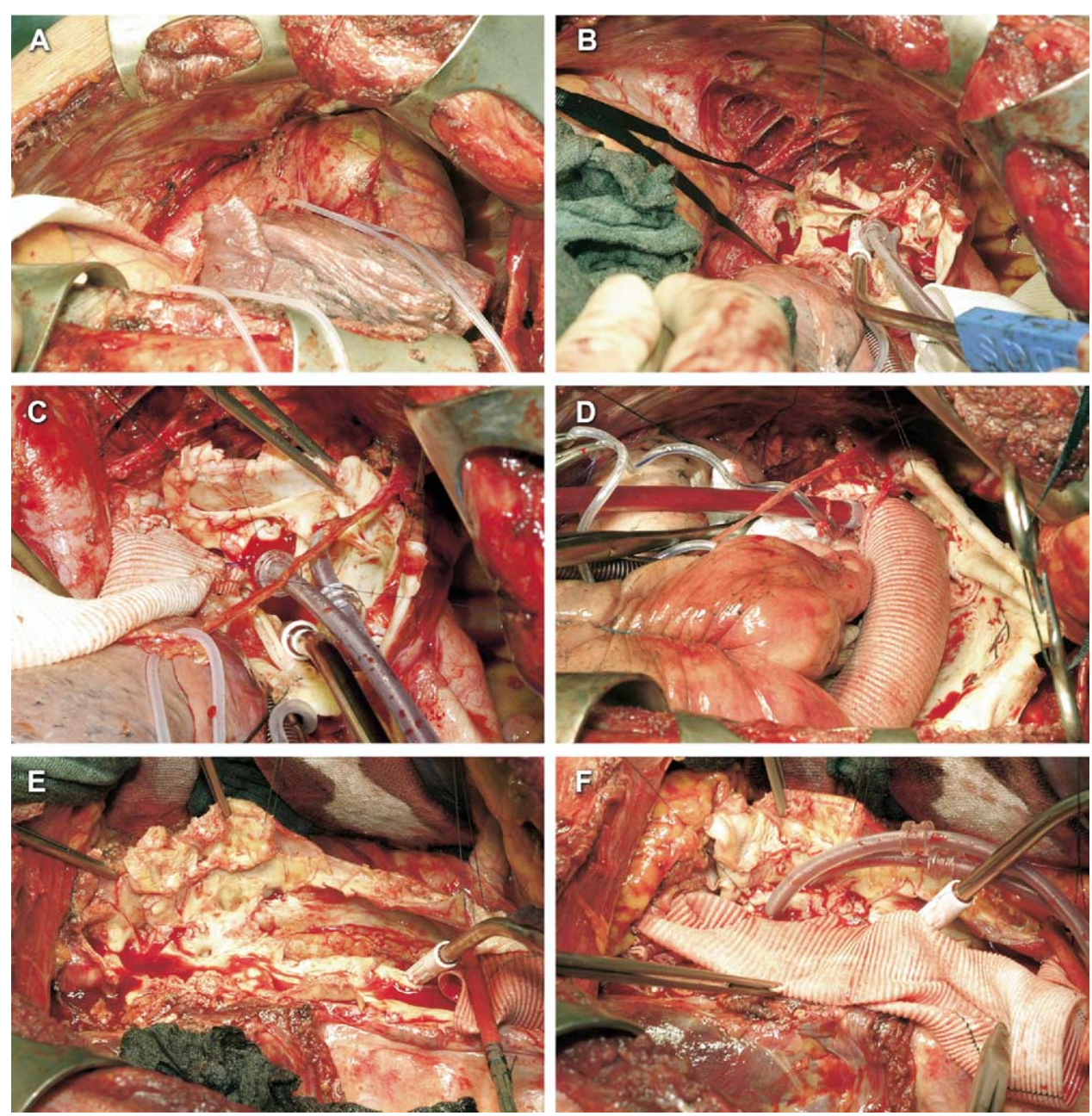

Figure E1. Intraoperative situs. The aortic arch was dissected (a), opened and selective cerebral perfusion was established via balloon tipped catheter in the brachiocephalic trunk and the left carotid artery (b). Following the proximal anastomosis between the old ascending aortic graft and the new prosthesis (c), the brachiocephalic trunk and the left carotid artery were reinserted as an island cuff. Retrograde perfusion of the upper body was reestablished via cannulation of the prosthesis (d). The complex dissection, the severity of calcifications and the contained rupture (area above the suction device) are shown in (e). During the distal anastomosis, abdominal organ perfusion was secured via balloon tipped catheters in the celiac trunk and superior mesenteric artery (f). 